\title{
Schulreformen in den USA - Trends, Nebeneffekte und Lektionen für Deutschland
}

\author{
Mit der PISA (Programme for International Student Assessment)-Studie wurde in \\ Deutschland vor knapp 15 Jahren eine Ära großer Schulreformen eingeleitet, die das ge- \\ samte Schulsystem nachhaltig auf Standardisierung, neue Strukturen der Rechenschafts- \\ legung sowie auf Testdaten- und Evidenzorientierung umstellten. Verbunden hiermit ist \\ bis heute die Hoffnung auf eine Erhöhung der Leistungsfähigkeit des Bildungssystems \\ und auf eine gleichzeitige Minimierung von Bildungsungleichheit. Um die Erfolgsaus- \\ sichten und langfristigen Folgen der Bildungsreformen besser abschätzen zu können, \\ wirft der Beitrag einen Blick in die USA, wo wesentlich längere Erfahrungen mit ähnli- \\ chen Steuerungsinstrumenten bestehen.
}

SIGRID HARTONG

\section{Einleitung}

Angeschoben durch eine grundlegende Ernüchterung über die mangelnde Leistungsfähigkeit des deutschen Bildungssystems im Rahmen des sogenannten „PISA-Schocks“ (hierzu aktuell Niemann 2014), haben Bildungspolitiker seit der Jahrtausendwende umfassend in die Qualitätssteigerung der deutschen Schulen investiert. Erklärtes Ziel war dabei, eine effektivere, besser auf den komplexen Systemcharakter von Bildung und Schule abgestimmte Qualitätskontrolle zu entwickeln (vgl. auch Hornberg/Parreira do Amaral 2012, S. 10). Um dies zu erreichen, initiierte die Kultusministerkonferenz (KMK) mit ihrer Handlungsagenda von 2002 tief greifende Reformen in Richtung Standardisierung sowie bessere Überwachung mittels entsprechender Datenerhebungen (monitoring) und testbasierter Rechenschaftslegungsmodelle (accountability und assessment). Bildungsforscher erarbeiteten binnen kurzer Zeit nationale Bildungsstandards und messbare Kompetenzmodelle. $\mathrm{Zu}$ den ergriffenen Maßnahmen gehörten auch die Gründung des Institutes für die Qualitätssicherung im Bildungswesen (IQB) und die Ausweitung zentral koordinierter Vergleichstests für Schulen. Gleichzeitig gab es Bestrebungen, die lokale und schulische Autonomie zu vergrößern und Bildungsprozesse damit lokal effizienter abzustimmen - etwa durch die Implementierung sogenannter Bildungsregionen. Insgesamt erhofften sich die Bildungspolitiker durch die
Einführung dieser „Large-Scale-Reformen“ (Gogolin et al. 2011) nicht nur eine umfassende Erhöhung der Leistung des deutschen Bildungssystems, sondern auch eine Verringerung der durch PISA laut angeprangerten Bildungsungleichheit. Gerade die Reformbefürworter brachten in diesem Zusammenhang immer wieder die Forderung nach einer Überwindung ideologischer Grabenkämpfe vor und setzten sich für die Etablierung einer kooperativen, expertenbasierten und durch Messbefunde abgesicherten Bildungssteuerung ein.

Bei allen Hoffnungen, die in diese radikalen, binnen kürzester Zeit durchgesetzten Bildungsreformen gesetzt wurden, ${ }^{1}$ sind nach mehr als einem Jahrzehnt nach ihrer Umsetzung jedoch zunehmend kritische Stimmen vernehmbar, ${ }^{2}$ die sowohl auf ein systematisches Ausbleiben der Steuerungsziele als auch auf äußerst problematische Nebenwirkungen hinweisen.

Im Kontext der wachsenden Kritik an den Auswirkungen der Bildungsreformen wirft dieser Beitrag einen Blick

1 Entsprechend wurde politisch vor allem der kontinuierliche Leistungszuwachs bei PISA nach 2003 als durchschlagender Erfolg der Reformen gewertet.

2 Prominente Beispiele vereinigter Bildungsreformkritiker sind hier etwa die im Jahre 2010 gegründete "Gesellschaft Bildung und Wissen" (GBW) oder das im letzten Jahr veröffentlichte Glossar „Ökonomisierung von Bildung" (GLOEB). 
in die USA, wo bemerkenswert lange Erfahrungen mit höchst unterschiedlichen Varianten standard-, test- und rechenschaftsbasierter Bildungssteuerung bestehen. Diese Varianten umfassten standardisierte, aber sanktionsschwache Tests ohne zentralisierte Überwachung (1950er Jahre), diffuse nationale Steuerungsziele (später Standards), gekoppelt mit sanktionsstarken staatlichen Tests (frühe 1990er Jahre), später ein national zentralisiertes, sanktionsstarkes Rechenschaftslegungssystem ohne übergeordnete Standards (zwischen 2001 und 2009) und zuletzt ein dem deutschen System sehr ähnliches Modell aus suprastaatlichen Standards und Tests mit größerer Freiheit für Lokalakteure (seit 2009).

Trotz dieser enormen Vielfalt an Steuerungsvarianten lassen sich dabei zwei übergeordnete Reformtrends identifizieren. Zum einen handelt es sich um eine ständig wachsende Evidenzorientierung. Damit ist die Herstellung wissenschaftlich „abgesicherten“ Wissens zur Wirkung von Bildungssteuerung und -reformen gemeint. Solches Wissen nimmt zunehmend die Gestalt technokratischer UrsacheWirkungs-Annahmen an, über die politische Interventionen in Bildung legitimiert werden. Zum anderen lässt sich ein genereller Trend zur Deregulierung im Sinne einer zunehmenden Verlagerung der Bildungssteuerung auf Wettbewerbe und externe Bildungsdienstleister feststellen. Beide Trends haben in den USA über die sehr unterschiedlichen Reformvarianten hinweg massive Dysfunktionalitäten erzeugt. Entsprechend, so wird im Folgenden gezeigt, mahnen gerade diese Langfristerkenntnisse aus den USA zu einer realistischeren Einschätzung möglicher Effekte der Schulreformen in Deutschland.

Abschnitt 2 erläutert zunächst genauer, weshalb gerade der Blick auf die US-amerikanische Bildungspolitik für den deutschen Reformzusammenhang so interessant erscheint. Im Anschluss hieran werden die zwei zuvor genannten Reformtrends - wachsende Evidenzorientierung und Deregulierung - inklusive ihrer Langfristeffekte in den USA näher betrachtet (3). Der darauf folgende Abschnitt stellt die Entwicklungen in den USA dem aktuellen Trend deutscher Bildungspolitik gegenüber und und diskutiert diese kritisch (4). Abschließend werden die zentralen Ergebnisse im Rahmen eines Fazits resümiert (5).

\section{Ein (aktualisierter) Blick in die USA}

Sowohl in Deutschland als auch in den USA trafen Versuche zur Durchsetzung zentral vereinheitlichender Bildungsreformen traditionell auf massiven Widerstand (vgl. Hartong 2015). Das Resultat waren dauerhaft zementierte Grabenkämpfe zwischen Gegnern und Befürwortern überstaatlicher Eingriffe. Diese spiegelten sich nicht zuletzt in einer starken Differenz zwischen einem „überlasteten“
Bildungsleitbild und der tatsächlichen Bildungspraxis wider, wobei Ersteres in Deutschland traditionell die Form des humboldtschen Bildungsideals zweckfreier Bildung annimmt, während es in den USA in der Betonung der individuellen Verantwortung des Lebenserfolgs (self-made man) besteht.

Im aktuellen deutschen Reformzusammenhang wurden die USA bislang vor allem in Bezug auf das dort unter der Bush-Administration Anfang des 21. Jahrhunderts implementierte Bildungsgesetz „No Child Left Behind“ (NCLB) debattiert: Unter dem erklärten Ziel, bis zum Jahr 2014 sämtliche Schüler zu einem ausreichend kompetenten Bildungsniveau zu bringen, schuf das Gesetz ein System zur bundesweit standardisierten Messung von Bildungsleistungen sowie zur Ermittlung des für jede Schule vorgesehenen „adäquaten jährlichen Leistungszuwachses“ (adequate yearly progress). Bei (Nicht-)Erreichen dieses normierten Leistungszuwachses etablierte das Gesetz ein radikales Sanktionssystem. Kaum eine Schule war jedoch in der Lage, die geforderten Leistungen zu erbringen. Stattdessen sahen sie sich plötzlich mit Konsequenzen des Mittelentzugs, der „erzwungenen“ Kooperation mit externen Experten und Dienstleistern oder sogar mit drohender Schließung konfrontiert. Da die Tests jedoch von den Staaten selbst entwickelt werden sollten und verbindliche nationale Standards fehlten, reagierten diese schließlich mit einem entsprechenden Absenken des Testniveaus, die Schulen und Lehrer hingegen mit Betrug oder strategischem „Aussortieren“ der getesteten Schüler, um die Sanktionen zu umgehen. Im Ergebnis führte NCLB daher eher zu einer Absenkung als zu einer Anhebung des Leistungsniveaus. Zugleich kam es zur Vergrößerung der Ungleichheit sowohl zwischen lernstarken und -schwachen Schülern als auch zwischen den Schulen. Zu Recht werden derartige Gesetze in der aktuellen deutschen Reformdiskussion rundheraus abgelehnt (Maier 2010).

Bildungspolitiker in den USA haben jedoch bereits weit vor NCLB den Weg in Richtung test- und standardbasierter Schulsteuerung über umfangreiche „Gesamtreformen“ eingeschlagen (Koretz 2008). Die unterschiedlichen Reformwellen und -strategien (darunter NCLB) sind dabei im Rahmen übergeordneter Reformtrends zu verordnen: Schulsteuerung wurde sukzessive zentralisiert, was dazu führte, dass im traditionell lokal organisierten Schulsystem der USA zunächst Akteure des Distrikts, dann des Staates und seit den 1960er Jahren auch der nationalen Ebene an Einfluss gewannen. Jeder dieser Zentralisierungsschritte Reese (2013) zufolge im Prinzip schon seit den 1840er Jahren - wurde von einer wachsenden Legitimierung externer Qualitätsstandards im Sinne vermessungsbasierter Steuerungsevidenzen als abgesichertes Wissen begleitet. $\mathrm{Zu}$ den hierdurch erzielten Effekten gehörten jedoch nicht nur die sukzessive Professionalisierung von Bildungsverwaltung und -kontrolle, sondern auch der wachsende Einfluss bildungsexterner Akteure. Dabei handelt es sich vor allem um Akteure der Testindustrie, deren Aufgabe primär in 
der Bereitstellung von Vermessungssystemen, Zahlen, und „Evidenzen“ besteht (auch Mehta 2013).

Das NCLB-Gesetz ist also nur eine der radikaleren von vielen Reformvarianten, auf die auch in den USA selbst eine massive Welle der Kritik folgte. Vor dem Hintergrund dieser Reformernüchterung gelang es einer Allianz aus Staats-, Beratungs- und Testakteuren nach 2009, mit den Common Core State Standards (CCSS) ein neues System zentralisierter Bildungsstandards zu etablieren. ${ }^{3}$ Diese wurden als eine „kooperativ initiierte“ und durch wissenschaftliche Evidenz abgesicherte Maßnahme präsentiert, die zugleich für die Überwindung politischer Grabenkämpfe stehen sollte. Neue, kognitionspsychologisch optimierte Systeme der Leistungsmessung - nun „Assessment" statt Test genannt - folgten kurze Zeit später. Zugleich erhielten die Staaten und Einzelschulen als Reaktion auf die Forderung nach einer Wiederherstellung dezentraler Steuerung ein über die NCLBRegelung hinausgehendes Maß an Autonomie. ${ }^{4}$ Dieser jüngste Trend ist dabei der Grundausrichtung der Reformen in Deutschland sehr ähnlich.

Umso wichtiger erscheint es, dass die USA aktuell, konkret seit 2014, mit einer neuen Welle der Reformernüchterung konfrontiert sind. Deutlicher denn je können die Kritiker heute zum einen zeigen, wie widersprüchlich die Effekte evidenzbasierter und deregulierter Steuerung - trotz der neuen Standards und erweiterten lokalen Autonomie - sind. Zum anderen weisen sie darauf hin, dass sich diese Effekte am Ende über die Reformvarianten hinweg manifestieren.

\section{Evidenzorientierung und}

\section{Deregulierung - zwei problematische Trends der US-Schulpolitik}

Die oben nur kurz erwähnten beiden Entwicklungstrends werden im Folgenden näher erläutert und im Hinblick auf ihre (Neben-)Effekte untersucht. Dabei handelt es sich um die wachsende Bedeutung einer (sanktionsgestützten) Evidenzorientierung (3.1) und das im Zuge staatlicher Deregulierung erzeugte Fehlen politischer Kontrollen gegenüber neuen Bildungssteuerungsakteuren (3.2). Beide Trends gehen mit einer umfassenden Neuverteilung öffentlicher Gelder einher.

\subsection{Die wachsende Verlagerung auf zentralisiert erzeugte Evidenzen}

Seit mehr als 25 Jahren wird im Zuge der unterschiedlichen Bildungsreformvarianten in den USA versucht, Bildungspolitik und Praxis stärker an wissenschaftlicher, durch Experten zertifizierter Evidenz auszurichten. Jede Form der Standardisierung und Zentralisierung ging infolgedessen mit einer schrittweisen, seit Inkrafttreten des NCLB-Geset- zes endgültig durchgesetzten „Verordnung von Evidenz" auf sämtlichen bildungspolitischen Ebenen einher. Hieran änderte auch die Tatsache nichts, dass das Ziel der Bewahrung oder Wiederherstellung lokaler Autonomie später wieder stärker ins Zentrum der Reformprogrammatik rückte. Eine ganz entscheidende Maßnahme war in diesem Zusammenhang die Organisation und Finanzierung sogenannter Unterstützungssysteme - vor allem in Form der systematischen Einbindung externer Experten in Lokalpolitik und Schulpraxis.

Bildungspolitiker nutzen hierbei Begriffe wie Benchmarks oder Best Practices, um herauszustellen, dass nachgewiesen ,erfolgreiche“ Bildungssteuerung modellhaft - das heißt auch jenseits lokaler Besonderheiten - übernommen werden kann. Die die Arbeitsweise der lokalen Schulgremien bestimmenden Grabenkämpfe wurden gleichzeitig als bildungspolitische Entscheidungsmaxime immer weniger akzeptiert und sollten im Zuge der Reformen - etwa über NCLB oder später über die CCSS - vollständig überwunden werden (McDonnell/Weatherford 2013, S. 491). Besonders in der Phase nach 2009 wurde Bildungssteuerung deshalb zunehmend von der lokalen Ebene losgelöst und (mitunter bildungsexternen) Expertengremien anvertraut, die im Fall der CCSS beispielsweise über „internationalen Wettbewerbsanschluss" verfügen mussten. ${ }^{5}$

Die Durchsetzung der Reformen erfolgte im Wesentlichen über die Ausschreibung von Wettbewerben um zentralpolitische Finanzmittel: Kein Staat, kein Distrikt musste im Sinne der Experten reformieren; ohne die freiwillige Teilnahme am Wettbewerb gab es aber auch kein Geld (Hartong 2015). Im Sinne der hiermit verfolgten Entpolitisierung wurden immer mehr Bundesmittel gezielt zur wissenschaftlichen Entwicklung verbesserter Tests oder Lernsysteme (etwa Online-Assessments oder digitales Lernen) sowie zur Schaffung eines Systems zur Messbarmachung von Bildung verwendet. Vor allem der Vergleich zwischen der NCLB- und der CCSS-Reform zeigt dabei, dass die Lokalpolitik im Zeitverlauf immer stärker mit Zwängen zur kostspieligen Evidenzgenerierung konfrontiert wurde -, die Weiss et al. (2008) zufolge entweder direkt oder indirekt mit Sanktionen behaftet war -, während sowohl die Legitimität lokalpolitischer Interessens-

3 www.corestandards.org.

4 Die Entlassung aus Sanktionen bzw. die Rückgabe von Autonomie erfolgte hierbei durch sogenannte WaiverProgramme, mit denen sich Einzelstaaten auf gewisse Reformkurse festlegen und hierfür entsprechende operative Freiheiten und Zeit für die Umsetzung erhalten.

5 Hiermit ist gemeint, dass die CCSS explizit als Modell glo bal angepasster, wettbewerbsfähiger Kompetenzen für College und (internationale) Karriere entwickelt wurden. Entsprechend waren es vor allem nationale, Nicht-Regierungs- und Beratungs- bzw. Testakteure, die die Standards auf den Markt brachten. 
verhandlung als auch die Mittel für infrastrukturelle Ressourcen zunehmend eingeschränkt wurden.

Die Lokalpolitik lässt sich jedoch nicht so einfach ausschalten. Tatsächlich reagieren die Lokalakteure auf die Einführung evidenzbasierter Steuerungsformen immer wieder aufs Neue mit offenem Widerstand, dem gleichzeitigen Auftreten von formaler Reform und einem Festhalten an traditionellen Praktiken sowie mit Betrug und Opportunismus. So zogen sich, obwohl die Sanktionen des NCLB-Systems zu diesem Zeitpunkt deutlich entschärft worden waren, viele Staaten seit 2014 spontan und heftig aus der Umsetzung der CCSS und entsprechender Tests zurück oder überschrieben bereits existierende Standards lediglich mit einem neuen „Label“.

Immer wieder zeigen die Reformprobleme in den USA die tatsächliche Unhintergehbarkeit der Lokalpolitik, da diese die Reformen - auch und vor allem über zähe Interessensverhandlungen - umsetzen, Leistungsdaten produzieren und dabei entsprechende Bildungsbudgets und -infrastrukturen bereitstellen müssen. Die bisherigen Versuche, Bildung politisch „neutral“ über ein gigantisches System externer Vermessungen und Evidenzgenerierung zu steuern, erzeugten daher immer dann Dysfunktionalitäten, wenn die Lokalpolitik in die Abhängigkeit von Sanktionen, Experten oder beidem getrieben wurde. Dysfunktionale Effekte wurden auch besonders dann sichtbar, wenn föderale/ staatliche Gelder ausschließlich in Sanktionen oder vermessende Expertentätigkeiten geleitet wurden, ohne die politischen und schulpraktischen Ressourcen für eine erfolgreiche Umsetzung der Reform zu finanzieren.

\subsection{Die fehlende Regulierung neuer Steuerungsakteure}

Ein entscheidender Punkt bei der Umsetzung der Bildungsreformen in den USA war immer wieder eine wachsende Forderung nach Transparenz. Nicht zufriedenstellende Testergebnisse erzeugten systematisches Misstrauen in lokale Akteure (allem voran in Lehrkräfte) und ihre Reformkapazitäten. Während NCLB mit massiven Sanktionen auf eine Erhöhung des Reformwillens setzte, lag der Schwerpunkt nach 2009 eher auf der umfassenden Implementierung effektiver Unterstützungssysteme. Was bislang nicht umgesetzt wurde, war hingegen eine systematische Erhöhung lokaler Ressourcen (siehe 3.1). Diese Tatsache ist durch den Umstand bedingt, dass die Bildungsreformen in den USA bereits seit Ende des 20. Jahrhunderts Teil einer umfassenden Sparpolitik waren (Fabricant/Fine 2013), die mehr und mehr auf finanzielle Auslagerung und auf Einbindung nichtöffentlicher Drittakteure setzte. Durch die umfassende Implementierung von Wettbewerben, so die Logik, sollten die Transparenz der schulischen Praxis erhöht und (Quasi-) Märkte geschaffen werden, auf denen sich die qualitativ hochwertigsten Modelle durchsetzen.

In den USA hat die Privatisierung der Bildung eine lange Tradition. Die NCLB-Reform hat jedoch eine neue Form der Privatisierung hervorgebracht, die von Patricia Burch (2009) auch als „neue Bildungsindustrie“ beschrieben wird. Während ursprünglich vorwiegend nicht-essenzielle Teile der Organisation von Schulbildung (etwa Transport, Schulessen, Gebäude etc.) in die Privatwirtschaft ausgelagert worden waren, wurden seit den 2000er Jahren auch Kernaspekte der Schulbildung in Marktgüter transformiert - darunter die Organisation ganzer Schuldistrikte, das Einstellen und Beaufsichtigen von Lehrkräften, die Sammlung und Aufbereitung von Schul- und insbesondere Testdaten, umfassende Beratungen, das Anbieten von Lernservices oder das Management von Bildung über IT-Systeme und -programme (Hard- und Software, technischer Support oder das Erstellen eines digitalisierten Curriculums).

Während die Lokalakteure der Bildungsverwaltung und -praxis zunehmend reguliert wurden (unter anderem auch über die Verpflichtung, die neuen Märkte in Anspruch zu nehmen oder standardisierte, onlinebasierte Tests durchführen zu müssen), fehlt bis heute genau diese regulierte Transparenz für eine Kontrolle der Bildungsindustrie. In den USA haben sich die in diesem Bereich neu entstandenen Märkte dabei auf undurchsichtige Art und Weise mit Stiftungs- und philanthropischen Aktivitäten vermischt, unter anderem im Bereich der wachsenden Anzahl sogenannter Vertragsschulen (Charter Schools) oder Bildungsmanagementorganisationen.

Auf dem komplexen Markt der neuen Bildungsindustrie fehlen nicht nur Preisregulierungen, sondern auch Qualitätsstandards oder Rechenschaftslegungszwänge. So ist unter anderem noch ungeklärt, wem eigentlich (sensible) Schuldaten gehören, wenn sie von einem externen Anbieter koordiniert und gesammelt werden, oder wie im Falle von Insolvenzen zu verfahren ist. Regelmäßig werden dabei skandalöse Praktiken und Geschäftsmodelle der neuen Marktakteure entdeckt - und doch lässt sich eine Re-Regulierung nur schwer durchsetzen, sobald sich erst einmal mächtige Allianzen der Liberalisierung etabliert haben.

Während die Marktakteure die neue Steuerung somit gezielt für sich nutzen, geraten die Lokalakteure zunehmend in eine unterlegene, wenn nicht gar abhängige Position allen Forderungen nach Transparenz zum Trotz. Im Gegenteil erscheinen die Ziele Transparenz, Qualitätssicherung und Kapazitätserhöhung immer dann in Gefahr, wenn profitorientierte Investoren motiviert werden sollen, langfristig Gelder zu investieren. Es ist wenig erstaunlich, dass die neue Bildungssteuerung damit mit dem typischen Dilemma der Regulation von Finanzmärkten konfrontiert ist. Nur zahlen den Preis hier nicht Anleger oder Steuerzahler, sondern Schüler und Lehrkräfte sowie (bildungsferne, wenig liquide) Eltern, die der Rolle strategisch wählender Konsumenten in vielen Fällen nicht entsprechen können.

Die im Zuge der Schaffung neuer Bildungsmärkte stattfindende Verlagerung der Bildungssteuerung auf Marktakteure ist mit der Absicht deutlicher Kostenersparnis einerseits und mit der Hoffnung auf die Verbesserung von Effizienz und Qualität von Bildung andererseits ver- 
knüpft. Gerade der Reformtrend aus den USA zeigt jedoch, dass Bildungspolitik dabei langfristig in ein Dilemma eigentlich notwendiger Regulierung bei gleichzeitig wachsender Abhängigkeit von den neuen Marktakteuren gerät. Gerade eine Kombination aus starker Regulierung/Rechenschaftspflicht für Lokalakteure und keiner oder einer nur geringen Regulierung der Drittanbieter führt dazu, dass Letztere vor allem ihrer eigenen Logik (Markterhalt, Profitgenerierung, kurzfristige Erfolgszahlen) folgen und damit die Erreichung der Reformziele erschweren, wenn nicht gar torpedieren. Längerfristig birgt die Verlagerung der Bildungspolitik auf Teile der Bildungsindustrie die Gefahr einer Entdemokratisierung in sich. Mit der fortschreitenden Entfesselung der neuen Bildungsmärkte wird diese Gefahr immer sichtbarer (Hartong 2015).

\section{Tendenzen deutscher Bildungspolitik}

Die vorangegangenen Überlegungen sollen zwei grundlegende Problematiken illustrieren. Diese ergeben sich unmittelbar aus der Neuausrichtung der Bildungssteuerung auf Vermessung und Evidenzorientierung, Bemühungen zur Deregulierung sowie der Einführung marktgesteuerter Regulationsmechanismen. Oftmals laufen diese Elemente der Bildungsreformen zusammen, indem beispielsweise Drittanbieter mit dem Bereitstellen von Evidenz (zum Beispiel in Form von Test- oder Evaluationssystemen) beziehungsweise der Unterstützung von Lokalakteuren bei der effektiven Umsetzung von Reformen beauftragt werden.

Die Erfahrungen aus den USA zeigen, dass der Bundeshaushalt in abnehmendem Maße Mittel zur Grundfinanzierung infrastruktureller oder technischer Bildungsressourcen bereitstellt und dass die öffentlichen Gelder hin zu den mit der Evidenzgenerierung betrauten Akteuren sowie zur Bildungsindustrie insgesamt umgeschichtet werden. Die lokale Bildungspolitik und -praxis wird infolgedessen von den neuen Steuerungsformen überlagert. Dennoch bleibt Schulbildung letztendlich in lokalpolitischer Verantwortung (Distrikte und lokale Schulgremien), sodass hier am Ende immer stärkere Widersprüche oder sogar wechselseitige Blockaden zwischen nationalen/staatlichen und lokalen Akteuren entstehen.

In Deutschland ist die Steuerungsumstellung wesentlich jünger. Anders als in den USA haben deutsche Bundespolitiker bislang darauf verzichtet, Schulen oder Bundesländer mit schlechten Testwerten zu sanktionieren. Nichtsdestoweniger häufen sich inzwischen auch hier Hinweise auf eine langfristige Erzeugung ähnlicher Dysfunktionalitäten wie in den USA.

So haben die KMK und das Bundesministerium für Bildung und Forschung (BMBF) nach der Publikation der ernüchternden PISA-Studie damit begonnen, öffentliche Bildungsgelder gezielt in den Aufbau eines komplexen Systems der Evidenzgenerierung und Bildungsvermessung zu investieren (Hartong 2014). So argumentiert Hans Peter Klein ${ }^{6}$ in der Wirtschaftswoche von Oktober 2013 treffend, dass mit der neuen Fokussierung auf die Messung und systematische Optimierung von Schülerkompetenzen ein „heiliger Gral“ der neuen Generation empirischer Bildungsforscher aus der Taufe gehoben wurde, die seitdem mit hohen Millionenbeträgen zentralisierter BMBF- und KMK-Programme versorgt werden. Binnen der letzten 15 Jahre ist hieraus ein riesiger Sektor empirischer Bildungsforschung entstanden, der unter anderem die dauerhafte Sammlung, Aufbereitung und Bereitstellung von Bildungsdaten organisiert und sich gleichzeitig als Wissens- und Finanzierungskontrolleur (etwa bezüglich der Auswahl „förderwürdiger" wissenschaftlicher Modellprojekte) etabliert hat.

Gleichzeitig erscheinen auch in Deutschland ideologisch motivierte Debatten über Bildung nicht mehr im selben Maße legitim wie vor den Bildungsreformen. Die Einführung nationaler Bildungsstandards gilt in den meisten Bundesländern als Programm eines „kooperativen Staates“ (Gördel 2008), das von parteilichen Grabenkämpfen Abschied nehmen und stattdessen allein dem neutralen Ziel des Lernzuwachses von Schülern dienen soll. Selbst die aktuellen Schulstrukturreformen mit dem tendenziellen Ergebnis einer Reduktion der Schulformen auf zwei Typen $($ Gymnasium +1$)$ werden von den Bildungspolitikern in vielen Bundesländern als Hinweise auf einen großen Schulfrieden gefeiert. Langfristig passt dieser Trend zur Entideologisierung jedoch auch hierzulande nicht zu den knappen lokalpolitischen Budgets, den Parteidebatten oder Wahlzyklen, sodass es auch hier permanent zu Widersprüchen zwischen verschiedenen Steuerungslogiken kommt.

Dies gilt besonders, seit die Basisfinanzierung der Schulen grundlegend neu organisiert wurde. Dem Leitbild der „eigenverantwortlichen Schule“ gemäß sollen die Schulen mittels gezielter Deregulierung aus der staatlichen Umklammerung befreit und zugleich dem lokalen Wettbewerb mit anderen Schulen ausgesetzt werden. Die widersprüchlichen, positiven wie negativen Folgen der eigenverantwortlichen Schule werden seit Längerem diskutiert. Entscheidend ist jedoch, dass mit der Umstellung auf Wettbewerbe und Bildungsmärkte auch in Deutschland neue Regulationsmechanismen erzeugt wurden. So werden Schulen etwa finanziell gezwungen, sogenannte Public-Private-Partnerships mit privaten Drittanbietern einzugehen - etwa Anbieter von Dienstleistungen oder Bildungsmedien. Ähnlich wie in den USA werden diese auch in Deutschland bislang nicht hinreichend kontrolliert oder transparent gemacht: „Öffentlichprivate Partnerschaften werden derzeit weder systematisch erfasst noch inhaltlich geprüft, geschweige denn auf ihre Effekte hin analysiert“" (Gericke 2013).

6 Mitbegründer der reformkritischen Gesellschaft für Bildung und Wissen (GBW) 


\section{Schlussfolgerungen}

Vor dem Hintergrund des langen Erfahrungswissens mit standard-, testund rechenschaftsbasierter Schulsteuerung in den USA lassen sich für die aktuellen Reformprozesse in Deutschland wichtige Schlussfolgerungen ziehen. Das eigentliche Problem, so eine zentrale Erkenntnis, besteht weniger in der (häufig diskutierten) Detailgestaltung (etwa die Rolle oder der Umfang von Tests) als in den dahinterliegenden Trends neuer Bildungssteuerung. Die Tendenzen zu einer Verabsolutierung von Evidenzorientierung einerseits sowie zu einer weitreichenden Deregulierung andererseits lassen die (lokale) Bildungspolitik und -praxis zunehmend in Abhängigkeit von Experten und bildungsexternen Akteuren geraten. Hoffnungen, einen schnellen Bildungsfortschritt allein über das massive Anhäufen von Funktionswissen („what works") und die Etablierung umfassender Wettbewerbe erzielen zu können, greifen dabei jedoch zu kurz.

Aus den bisherigen Reformerfahrungen in den USA kann daher gefolgert werden, dass die Forderung nach Transparenz und Rechenschaftslegung dysfunktional wird, sobald bestimmte Akteure hiervon ausgenommen werden. Im Gegenteil muss es eine Pflicht der öffentlichen Hand bleiben, undurchsichtige Abhängigkeiten systematisch zu verhindern und im Zweifelsfall immer auf der Seite der Schulen zu stehen. Hierfür muss Letzteren vertraut und dieses Vertrauen öffentlich bekundet werden.

Weiter muss legitim und ausführlich darüber debattiert werden, dass die neue Bildungssteuerung bei Weitem keine „neutrale“, sondern vielmehr eine hochgradig politisierte, marktbasierte Steuerung ist, die gerade durch ihre Ummantelung mit einer „neutralen Aura der Lernorientierung“ weitreichende Macht entfalten kann. Gerade deswegen bleibt die politischideologische Debatte ein Kernaspekt der Bildungssteuerung und muss als solche (wieder) anerkannt werden. Dies hätte zur Folge, dass die (voraussetzungsreichen) normativen Elemente neuer Bildungssteuerung ausreichend demokratisch diskutiert werden.

Die Erfahrungen aus den USA zeigen, dass trotz politischer Versuche, ungewollte Effekte von Reformen über „Nachsteuerung“ auszubessern, diese nicht mehr oder nur sehr schwer beseitigt werden können. Dies gilt für einmal etablierte Kontrollstrukturen - etwa die Verwendung von Tests für Sanktionen - oder für die Verlagerung wichtiger Steuerungsfunktionen auf Bildungsmärkte - etwa für die Kontrolle von Schuldaten. Es muss daher von Anfang an systematisch verhindert werden, dass zu starke Einflussdifferenzen zwischen unregulierten Kontrolleuren und den von ihnen Kontrollierten entstehen.

In diesem Sinne sollten Finanzmittel nicht nur über Anreiz- oder evidenzbasierte Modelle verteilt werden, sondern es muss weiterhin eine solide Inputfinanzierung geben - mit entsprechendem Raum für eine eigenverantwortliche Umsetzung. Im Sinne von Peter M. Taubmans (2014) Plädoyer für „opportunity-to-study-standards“ wären Standards zu entwickeln, die, statt auf ein sich immer enger zuziehendes Messregime zu setzen, eine Sicherstellung von Infrastruktur garantieren - und von Zeit.

\section{LITERATUR}

Burch, P. (2009): Hidden markets. The new education privatization, New York Fabricant, M./Fine, M. (2013): The changing politics of education. Privatization and the dispossessed lives left behind, London

Gericke, C. (2013): Public Private Partnership, in: GLOEB. Glossar Ökonomisierung von Bildung, http://www.gloeb.de/index.php?title=Public_Private_ Partnership (letzter Zugriff: 10.04.2015)

Gogolin, I./Baumert, J./Scheunpflug, A. (2011): Transforming education. Umbau des Bildungswesens, in: Zeitschrift für Erziehungswissenschaft 13 (4), S. $529-547$

Gördel, B. (2008): Die Einführung der nationalen Bildungsstandards in drei Bundesländern - eine explorative Studie zu Implementationsstrategien, in: Langer, R: (Hrsg.): Warum tun die das? Governanceanalysen zum Steuerungshandeln in der Schulentwicklung, Wiesbaden, S. 193-220

Hartong, S. (2014): Neue Bildungsregulierung im Zeitalter der "governance by numbers". Das Beispiel standardbasierter Bildungsreform in Deutschland und den USA, in: Leviathan 42 (4), S. 1-29

Hartong, S. (2015): Global policy convergence through distributed governance? The emergence of 'national' education standards in the US and Germany, in: Journal of International and Comparative Social Policy 31 (1), S. $10-33$

Hornberg, S./Parreira do Amaral, M. (2012): Deregulierung im Bildungswesen, Münster

Klein, H. P. (2013): DerTestwahn der Bildungsforscher, in: Wirtschaftswoche vom 10. Oktober

Koretz, D. (2008): Measuring up. What educational testing really tells us, Cambridge

Maier, U. (2010): Effekte testbasierter Rechenschaftslegung auf Schule und Unterricht. Ist die internationale Befundlage auf Vergleichsarbeiten im deutschsprachigen Raum übertragbar?, in: Zeitschrift für Pädagogik 56 (1), S. 112-118 McDonnell, L./Weatherford, M. S. (2013): Organized interests and the common core, in: Educational Researcher 42 (9), S. 488-497

Mehta, J. (2013): The allure of order: High hopes, dashed expectations, and the troubled quest to remake American schooling, Oxford

Niemann, D. (2014): After the big bang - German education policy in the wake of the PISA study and the Bologna process, in: Martens, K./Knodel, P./Windzio. M. (Hrsg.): Internationalization of education policy. A new constellation of statehood in education?, Basingstoke, S. 91-114

Reese, W. J. (2013): Testing wars in the public school. A forgotten history, Cambridge

Taubmann, P. M. (2014): An alternative to education by decree, in: Journal of Curriculum and Pedagogy 11 (1), S. 46-49

Weiss, C. H./Murphy-Graham, E./Petrosino, A./Gandhi, Allison G. (2008): The fairy godmother - and her warts. Making the dream of evidence-based policy come true, in: American Journal of Evaluation 29 (1), S. 29-47

\section{AUTORIN}

SIGRID HARTONG, Dr. rer. pol., ist Wissenschaftliche Mitarbeiterin an der Helmut-Schmidt-Universität Hamburg. Arbeitsschwerpunkte: International und historisch vergleichende Bildungsforschung, Transnationalisierung von Bildung, qualitative Netzwerk- und Feldforschung.

hartongs@hsu-hh.de 